\title{
Development and internal validation of a nine-IncRNA prognostic signature for prediction of overall survival in colorectal cancer patients
}

\author{
Zhiqiao Zhang ${ }^{1}$, Qingbo Liu ${ }^{2}$, Peng Wang ${ }^{\text {Corresp.. }}{ }^{1}$, Jing Li ${ }^{1}$, Tingshan He ${ }^{1}$, Yanling Ouyang ${ }^{1}$, Yiyan Huang \\ 1 , Weidong Wang ${ }^{2}$ \\ 1 Department of Infectious Diseases, Shunde Hospital, Southern Medical University, Shunde District, Guangdong, China, Shunde, Guangdong, China \\ Corresponding Author: Peng Wang \\ Email address: sdgrxjbk@smu.edu.cn
}

Background. Colorectal cancer remains a serious public health problem due to the poor prognosis. In the present study, we attempted to develop and validate a prognostic signature to predict the individual mortality risk in colorectal cancer patients.

Materials and Methods. The original study datasets were downloaded from The Cancer Genome Atlas database. The present study finally included 424 colorectal cancer patients with wholly gene expression information and overall survival information.

Results. A nine-IncRNA prognostic signature was built through univariate and multivariate Cox proportional regression model. Time-dependent receiver operating characteristic curves in model cohort demonstrated that the Harrell's concordance indexes of nine-IncRNA prognostic signature were 0.768 (95\% Cl: 0.717-0.819), 0.778 (95\% Cl: 0.727-0.829) and 0.870 (95\% Cl: 0.819-0.921) for 1-year, 3-year and 5-year overall survival respectively. In validation cohort, the Harrell's concordance indexes of nineIncRNA prognostic signature were 0.761 (95\% Cl: 0.710-0.812), 0.801 (95\% Cl: 0.750-0.852) and 0.883 (95\% Cl: 0.832-0.934) for 1-year, 3-year and 5-year overall survival respectively. According to the median of nine-IncRNA prognostic signature score in model cohort, 424 CRC patients could be stratified into high risk group $(n=212)$ and low risk group $(n=212)$. Kaplan-Meier survival curves showed that the overall survival rate of high risk group was significantly lower than that of low risk group $(P<0.001)$.

Discussion. The present study developed and validated a nine-IncRNA prognostic signature for individual mortality risk assessment in colorectal cancer patients. This nine-IncRNA prognostic signature is helpful to evaluate the individual mortality risk and to improve the decision making of individualized treatments in colorectal cancer patients. 
1 Development and internal validation of a nine-IncRNA prognostic signature

2 for prediction of overall survival in colorectal cancer patients

Zhiqiao Zhang ${ }^{1 \#, ~ Q i n g b o ~ L i u ̈ " \#, ~ P e n g ~ W a n g ~}{ }^{1 *}$, Jing Li ${ }^{1}$, Tingshan $\mathrm{He}^{1}$, Yanling Ouyang ${ }^{1}$, Yiyan Huang ${ }^{1}$, Weidong Wang ${ }^{2}$

1 Department of Infectious Diseases, Shunde Hospital, Southern Medical University, Shunde District, Guangdong, China

2 Department of Hepatobiliary Surgery, Shunde Hospital, Southern Medical University, Shunde District, Guangdong, China

\# These authors contributed equally to this work.

* Corresponding author: Peng Wang (E-mail: wangpeng1962@yeah.net)

(1)




\section{Abstract}

Background. Colorectal cancer remains a serious public health problem due to the poor prognosis. In the present study, we attempted to develop and validate a prognostic signature to predict the individual mortality risk in colorectal cancer patients.

Materials and Methods. The original study datasets were downloaded from The Cancer Genome Atlas database. The present study finally included 424 colorectal cancer patients with total gene expression information and overall survival information.

Results. A nine-IncRNA prognostic signature was built through univariate and multivariate Cox proportional regression model. Time-dependent receiver operating characteristic curves in model cohort demonstrated that the Harrell's concordance indexes of nine-IncRNA prognostic signature were 0.768 (95\% Cl: $0.717-0.819), 0.778$ (95\% Cl: $0.727-0.829)$ and 0.870 (95\% Cl: 0.819-0.921) for 1-year, 3-year and 5-year overall survival respectively. In validation cohort, the Harrell's concordance indexes of nine-IncRNA prognostic signature were 0.761 (95\% Cl: $0.710-0.812), 0.801$ (95\% Cl: 0.750-0.852) and 0.883 (95\% Cl: 0.832-0.934) for 1-year, 3-year and 5-year overall survival respectively. According to the median of nine-IncRNA prognostic signature score in model cohort, 424 CRC patients could be stratified into high risk group $(n=212)$ and low risk group $(n=212)$. KaplanMeier survival curves showed that the overall survival rate of high risk group was significantly lower than that of low risk group $(P<0.001)$.

Discussion. The present study developed and validated a nine-IncRNA prognostic signature for individual mortality risk assessment in colorectal cancer patients. This nine-IncRNA prognostic signature is helpful to evaluate the individual mortality risk and to improve the decision making of individualized treatments in colorectal cancer patients. 
Colorectal cancer $(\mathrm{CRC})$ is one of the most common malignant tumors and one of the leading causes of cancer-related death, resulting in 135,430 estimated new cases and 50,260 estimated deaths in the United States in 2017 (Siegel et al. 2017; Siegel et al. 2016). The 5-year survival rates of CRC patients varied dramatically in patients with different tumor stages (Ferlay et al. 2010). The 5-year survival rate of localized CRC patients was $90 \%$, whereas it were $71 \%$ and $14 \%$ for CRC patients with regional metastasis and distant metastasis respectively (Siegel et al. 2017).It was reported that the 5year survival rate was approximately $10 \%$ in stage IV CRC patients (Sanoff et al. 2008). Therefore, it is of great importance to develop a reliable prognostic biomarker to predict the prognosis and optimize the clinical therapy decision. 
merely the prediction of overall survival for a special subgroup, but not the individual risk prediction of overall survival. Therefore, an ideal individual risk predictive model should be easy to obtain, calculate and understand for clinical application.

Several studies have used nomogram method to predict the prognosis of different cancers (Li et al. 2015; Tian et al. 2017). This method has two advantages in predicting the prognosis. Firstly, the nomogram is convenient to calculate and evaluate the individual probability of disease without complex formula. Secondly, this method provides a straightforward individual risk and the result is easy to understand for patients. Therefore, the nomogram method is suitable for prediction of prognosis. To the best of our knowledge, the present study is the first to develop a prognostic nomogram by using the IncRNA expression data for overall survival of CRC patients.

In the present study, our objective was to develop and validate a IncRNA-based prognostic signature to predict the overall survival of CRC patients. To improve the quality of prognostic model, the development and validation of the prognostic model in the present study were performed in accordance with the guidelines of Transparent Reporting of a multivariable prediction model for Individual Prognosis Or Diagnosis (TRIPOD) (Collins et al. 2015).

\section{MATERIALS AND METHODS}

\section{Study protocol approval}

The data download and analyses were performed according to the policies of The Cancer Genome Atlas (TCGA) database. Since the study datasets in the present study were all downloaded from TCGA database, additional ethics approval was not needed. All data collections and analyses were in accordance with the principles of Declaration of Helsinki.

\section{The gene expression dataset}

The original RNA sequencing dataset was obtained from GDC Data Portal (https://portal.gdc.cancer.gov/exploration). The dataset was downloaded in May 31, 2018. The RNA sequencing data were generated on the Illumina HiSeq 2000 RNA Sequencing platform. The 
103

104

105

106

107

108

109

110

111

112

113

114

115

116

\section{7}

118

119

120

121

122

123

124

125

126

127

128

129

downloaded RNA sequencing data contained the original gene read counts of 60,483 genes from 458 CRC tumor tissues and 41 non-tumor normal tissues. The duplicated samples were removed from the present study $(n=22)$. In the present study, the IncRNAs ID were downloaded from GENCODE Version 7 (Derrien et al. 2012). Finally the extracted gene expression data of 14,449 IncRNAs from 458 CRC tissues and 41 non-tumor normal tissues were selected for differentially expressed IncRNAs.

\section{Differential expression analyses}

The "edgeR" package was applied for the differential expression analyses and the original gene expression read counts was normalized by the Trimmed Mean of M (TMM) method (Robinson \& Oshlack 2010). The genes whose average expression read counts lower than 1 were filtered out from the present study. The F-tests was used for assessments of quasi-likelihood. Thresholds of differentially expressed IncRNAs were Padj $<0.05$ and |log2 fold change| $>2$.

\section{Heat map and volcano map}

The heat map and volcano map were drawn according to the normalized gene expression values of differentially expressed IncRNAs. The darker color represented higher expression level of differentially expressed IncRNA.

\section{The model cohort}

The clinical dataset of 629 CRC patients were downloaded from cBioPortal database (May 31, 2018, http://www.cbioportal.org/data_sets.jsp). The patients with overall survival time less than one month were excluded from the present study to avoid the impact of unrelated causes of death $(n=49)$. The clinical dataset was matched to the gene expression dataset. The patients without gene expression information were excluded from the present study $(n=156)$. Finally 424 CRC patients were enrolled for further survival analysis. Figure 1 was the study flowchart for patient selection in the present study. The end-point in the present study was overall survival (OS). The average follow-up time was 
130

131

132

133

134

135

136

137

138

139

140

141

142

143

144

145

146

147

148

149

150

151

152

153

154

155

156

$30.0 \pm 25.5$ months. Overall survival time was calculated from initial diagnosis time to death time or the last follow-up time. The maximum value and the minimum value of overall survival time were 140.9 months and 1.0 month. The study time was from May 18, 2010 to January 27, 2015. The missing data in the present study were coded as "NA" in all tables and analyses.

\section{Assessment of predictive performance}

The Harrell's concordance index (C-index), calibration plot and Time-dependent receiver operating characteristic (ROC) curves were used to assess the predictive performance of prognostic signatures.

The predictive performance of prognostic signature in the current study was compared with two previous prognostic signatures (named RS IncRNA score and Risk score). The formulas of RS IncRNA score and Risk score were as follows:

RS IncRNA score $=(0.1337 \times$ expression value of RP1-170019.17) $+(0.0633 \times$ expression value of RP11-785D18.3) + (0.8131 $\times$ expression value of RP11-798K3.2)+ (-1.5194× expression value of XXbac-B476C20.9) $+(4.3132 \times$ expression value of RP11-481J13.1) $+(0.4497 \times$ expression value of RP11-167H9.4 ) (Fan \& Liu 2018). In the formula of RS IncRNA score, the IncRNA expression values were normalized by "DEseq" packages.

Risk score $=\exp _{\text {LINC01555 }}{ }^{*}(-0.191)+\exp _{\text {RP11-108K3.1 }}{ }^{*}(0.318)+\exp _{\text {LINC01207 }}{ }^{*}(-0.191)+\exp _{\text {RP11-610P16.1 }}{ }^{*}(-$ 0.163) (Zeng et al. 2017). In the formula of Risk score, the IncRNA expression values were $\log _{2^{-}}$ transformed.

\section{Internal validation by using bootstrap resampling method}

The bootstrap resampling method has been recommended for internal validation of the predictive model (Blackstone 2001; Grunkemeier \& Wu 2004). The validation cohort in the present study was constructed by drawing $424 \mathrm{CRC}$ patients with replacements from the original model cohort.

\section{Statistical analysis}

The statistical analyses were carried out by using the R software (version 3.4.1) and SPSS Statistics 
157

158

159

160

161

162

163

164

165

166

167

168

169

170

171

172

173

174

175

176

177

178

179

180

181

182

183

19.0 (SPSS Inc., an IBM Company). The following R packages were carried out as needed in the current study: "survival", "rms", "pROC", "plyr", "glmnet", and "timeROC". Continuous variables in the present study were presented as mean \pm standard deviation. Continuous variables were compared by t-test or Mann-Whitney U test. Categorical variables were compared by chi-squared test or Fisher's exact test. A 2-tail $P<0.05$ was defined as statistically significant in the present study.

\section{RESULTS}

\section{Study cohorts}

The present study finally included 424 CRC patients with total IncRNA expression information and overall survival information. The average age was $66.7 \pm 13.0$ years and the average overall survival time was $30.0 \pm 25.5$ months in model cohort. There were $102(24.0 \%)$ patients died during the followup period in model cohort, whereas there were 108 (25.5\%) patients died during the follow-up period in validation cohort. The clinical characteristics of CRC patients in model cohort and validation cohort were summarized in Table 1. There were no significant differences in terms of clinical characteristics and IncRNA expression between model cohort and validation cohort. There were no missing data in terms of survival status, survival time and IncRNA expression value.

\section{Differentially expressed analyses}

We performed differential expression analyses by comparing all gene expression values between 458 tumor tissues and 41 normal colon tissues. The "edgeR" package identified 574 differentially expressed genes for overall survival. The heat map and volcano map of differential expression genes were presented in supplementary Figure 1 and Figure 2 respectively.

\section{Construction of prognostic signature for overall survival}

The univariate Cox regression analysis was performed to explore the potential prognostic IncRNAs for overall survival. The univariate Cox proportional regression analysis identified 33 potential IncRNA predictors for overall survival. Using multivariate Cox proportional regression analysis, a nine-IncRNA 
184

185

186

187

188

189

190

191

192

193

194

195

196

197

198

199

200

201

202

203

204

205

206

207

208

209

210

prognostic signature (Fig.2) was constructed based on the potential prognostic IncRNA predictors determined by Cox regression analysis. The overall information of nine prognostic IncRNA predictors were summarized in Table 2. The formula of nine-IncRNA prognostic signature was as follows: nineIncRNA prognostic signature score $=\left(0.837^{*} \mathrm{RP} 11 \_815 \mathrm{M} 8.1\right)+\left(0.822^{*} \mathrm{RP} 11 \_342 \mathrm{~A} 23.2\right)+\left(0.905^{*}\right.$ RP11_264B14.1)+(-0.529* AC064834.1)+(0.907* RP11_108K3.2)+(-0.745* LINC01571)+(1.241* RP11_383I23.2)+(-0.737*AC079612.1)+(0.725*AC005256.1).

\section{Performance of nine-IncRNA prognostic signature in model cohort}

The nine-IncRNA prognostic signature score were generated according to the previous formula. The distributions of nine-IncRNA prognostic signature score (Fig.3A), overall survival status and overall survival time (Fig.3B) in model cohort were shown in Figure 3. In model cohort, the Harrell's concordance-index (C-index) of nine-IncRNA prognostic signature was 0.757 (95\% Cl: 0.706-0.808).

\section{Kaplan-Meier survival curves and log-rank test in model cohort}

The median of nine-IncRNA prognostic signature score was used as cutoff value to stratify CRC patients into high risk group and low risk group. Kaplan-Meier survival curves (Fig.4) and log-rank test were used to compare the difference of overall survival rate between high risk group and low risk group. As shown in Figure 4, patients with high nine-IncRNA prognostic signature score had poorer overall survival rate than patients with low nine-IncRNA prognostic signature score $(P<0.001)$.

\section{Time-dependent receiver operating characteristic curves in model cohort}

We further explored the predictive accuracy of nine-IncRNA prognostic signature score compared with two previous prognostic signatures by using time-dependent receiver operating characteristic curves (Fig.5). The C-indexes of nine-IncRNA prognostic signature, RS IncRNA score and Risk score were $0.768,0.654$ and 0.658 for 1-year overall survival (Fig.5A) respectively, whereas it were 0.778 , 0.666 and 0.582 for 3-year overall survival (Fig.5B). For 5-year overall survival (Fig.5C), the Cindexes of nine-IncRNA prognostic signature, RS IncRNA score and Risk score were 0.870,0.681 and 
2110.633 respectively.

212

\section{Calibration curves in model cohort}

214 The calibration curves were used to assess the predictive performance of nine-IncRNA prognostic

215 signature. The calibration curves for 1-year (Fig.5D), 3-year (Fig.5E) and 5-year (Fig.5F) overall

216 survival demonstrated that there were a good agreement between the predictive probability and the

217 actual overall survival in model cohort.

218

Internal validation of nine-IncRNA prognostic signature

The validation cohort $(n=424)$ was generated by random drawing from the model cohort with replacement method. The nine-IncRNA prognostic signature score were generated according to the previous formula for patients in validation cohort. The distributions of nine-IncRNA prognostic signature score (Fig.6A), overall survival status and overall survival time (Fig.6B) in validation cohort were shown in Figure 6 . The C-index of nine-IncRNA prognostic signature was $0.75195 \% \mathrm{Cl}: 0.700-$ 0.802) in validation cohort.

\section{Kaplan-Meier survival curves and log-rank test in validation cohort}

The previous cutoff value of nine-IncRNA prognostic signature score in model cohort was used as the cutoff value to stratify CRC patients into high risk group and low risk group in validation cohort. As shown in Figure 7, the log-rank test indicated that the overall survival rate in high risk group was significantly lower than that in low risk group $(P<0.001)$.

\section{Time-dependent receiver operating characteristic curves in validation cohort}

In validation cohort, the C-indexes of nine-IncRNA prognostic signature, RS IncRNA score and Risk score were $0.761,0.695$ and 0.664 for 1-year overall survival (Fig.8A) respectively, whereas it were $0.801,0.660$ and 0.582 for 3-year overall survival (Fig.8B). For 5-year overall survival (Fig.8C), the C- 
238

239

240

241

242

243

244

245

246

247

248

249

250

251

252

253

254

255

256

257

258

259

260

261

262

263

264

0.616 respectively.

\section{Calibration curves in validation cohort}

The calibration curves for 1-year (Fig.8D), 3-year (Fig.8E) and 5-year (Fig.8F) overall survival indicated a good agreement between the predictive probability of overall survival and the actual overall survival in validation cohort.

\section{Independence of nine-IncRNA prognostic signature for overall survival}

We further carried out multivariate Cox regression analyses to explore whether nine-IncRNA prognostic signature was an independent influence factor for overall survival in model cohort and validation cohort. After adjustment of other clinical variables, including age, gender, the American Joint Committee on Cancer (AJCC) PT, AJCC PN, AJCC PM and AJCC stage (Table 3), the results of multivariate Cox regression analysis indicated that the nine-IncRNA prognostic signature was an independent influence factor for overall survival of CRC patients.

\section{Functional enrichment analysis of prognostic signature}

We performed functional enrichment analysis to explore the biological pathway and process correlated with this nine-IncRNA prognostic signature. At first, the pearson correlation coefficients between these prognostic IncRNA expression values and the mRNA expression values were calculated in the TCGA dataset. Then the genes correlated with at least one of these prognostic IncRNAs (defined as |Pearson correlation coefficient| > 0.5) were included into the following functional enrichment analysis. The gene ontology (GO) biological process enrichment analysis and Kyoto Encyclopedia of Genes and Genomes (KEGG) signaling pathway analysis were presented in Figure 9 by using the above identified genes in the Database for Annotation, Visualization, and Integrated Discovery (DAVID) (https://david.ncifcrf.gov/) Bioinformatics Resources. The results of functional enrichment analysis demonstrated that the co-expressed genes were mainly enriched in Gprotein coupled receptor signaling pathway, potassium ion transmembrane transport, carboxylic acid 
265

266

267

268

269

270

271

272

273

274

275

276

277

278

279

280

281

282

283

284

285

286

287

metabolic process response to hormone, regulation of ion transmembrane transport (Figure.9).

\section{DISCUSSION}

In the present study, we developed and validated a nine-IncRNA prognostic signature that was helpful for individual mortality risk prediction and survival stratification of CRC patients. This nineIncRNA prognostic signature was helpful for patients to ascertain their individual mortality risk and optimize their personalized treatment strategies. Time-dependent receiver operating characteristic curves demonstrated that this nine-IncRNA prognostic signature was superior to other two previous prognostic signatures for prediction of overall survival.

This nine-IncRNA prognostic signature without pathological parameters provided a noninvasive preoperative prediction method for overall survival of CRC patients. Firstly, this nine-IncRNA prognostic signature could provide a preoperative individual mortality risk prediction, which was helpful for patients without medical knowledge to understand the actual individual mortality risk in different clinical endpoints. Secondly, the median of nine-IncRNA prognostic signature score was used as the cutoff value to stratify colorectal patients into high risk group and low risk group. The Kaplan-Meier survival curves demonstrated that the overall survival rate in high risk group was significantly lower than that in low risk group. Therefore, this nine-IncRNA prognostic signature was helpful for patients with high mortality risk to make clinical decision of receiving active individualized treatment.

As reported in the original article, the AUROCs of Risk score for 5-year overall survival were 0.706 and 0.619 in model group and validation group respectively (Zeng et al. 2017). The AUROCs of RS IncRNA score were 0.731 and 0.727 in training dataset and testing dataset (Fan \& Liu 2018). The AUROCs of Risk score and RS IncRNA score in the present study were lower than that in original articles. The differences of AUROC might be caused by the following reasons: Firstly, the sample size in the present study was 424 , whereas it were 371 for Risk score and 568 for RS IncRNA score. The difference of sample size would result in an influence to the predictive accuracy of prognostic models. Secondly, the numbers of selected IncRNAs were different in three prognostic signatures. The 
292 number of selected IncRNAs in nine-IncRNA prognostic signature was 9, whereas it were 4 for Risk

293 score and 6 for RS IncRNA score. Thirdly, Risk score was calculated by using the IncRNA expression

294 values which have been $\log _{2}$-transformed. RS IncRNA score was calculated by using the IncRNA

295 expression values which have been normalized by "DEseq" package. In order to improve the

universal applicability of the research results, the medians of original IncRNA expression values were

used as cutoff values to stratify IncRNA expression values into high expression group (as value 1)

and low expression group (as value 0) in the present study. The nine-IncRNA prognostic signature score were calculated by using the binary IncRNA expression values according to the medians of original IncRNA expression values. This adjustment was helpful to improve the clinical application of prognostic model in other study population.

The present study has the following advantages: First, this nine-IncRNA prognostic signature can be used to assess the individual mortality risk through simple chart by patients. Second, this nineIncRNA prognostic signature can provide an individual mortality risk assessment for 1-year, 3-year and 5-year overall survival. Individual mortality risk prediction at different time points is of great significance to persuade CRC patients receive a timely differentiated intensive treatment. Third, this nine-IncRNA prognostic signature provides individual mortality risk probability and the result is easy to understand for patients without medical knowledge. Fourth, this nine-IncRNA prognostic signature can serve as a preoperative non-invasive prediction method for overall survival of CRC patients.

310 Therefore, this nine-IncRNA prognostic signature is suitable for preoperative prediction of overall survival, especially for advanced CRC patients who can't tolerate surgery.

312 The current study has two shortcomings which must be considered for interpreting the clinical significance of the results. First, the current study constructed a prognostic signature by using nine

314 lncRNA predictors. However, due to the different gene detection platforms and different IncRNA 315 name, we could not obtain second independent dataset containing these nine IncRNA predictors in 316 other databases including Gene Expression Omnibus (GEO) database, ArrayExpress database, and

317 The Atlas of Noncoding RNAs in Cancer (TANRIC). We performed an internal validation by using 318 bootstrap resampling method but not external validation by using independent data. Therefore, this 
319 nine-IncRNA prognostic signature in the current study need further external validation through

320 external independent dataset. Second, we searched TCGA database and ascertained nine IncRNAs

321 as prognostic predictors for overall survival in CRC patients. The relationship between these IncRNA

322 predictors and the prognosis of CRC patients was not clear. Third, the average follow-up time was

$32330.0 \pm 25.5$ months and there were only $102(24.0 \%)$ patients died during the follow-up period in model

324 cohort. Taking into account the limited follow-up time and relatively small event size, the association

325 between these IncRNA predictors and the prognosis of CRC patients should be validated in an

326 additional cohort with longer follow-up period and larger event size. Therefore, large prospective

327 clinical studies are needed to clarify the relationship between these IncRNA predictors and the

328 prognosis of $\mathrm{CRC}$ patients.

329

330

\section{CONCLUSIONS}

331 In summary, the present study developed and validated a nine-IncRNA prognostic signature for 332 individual mortality risk assessment in colorectal cancer patients. This nine-IncRNA prognostic 333 signature is helpful to determine the individual mortality risk of overall survival and to improve the 334 decision making of individualized treatments in colorectal cancer patients.

336 Abbreviations

337 CRC Colorectal cancer

338 AUC Area under the ROC curve

339 OS Overall survival

340 ROC Receiver operating characteristic curve

341 LncRNA Long non-coding RNA

342 TCGA The Cancer Genome Atlas

343 RPKM Reads per kilobase per million mapped reads

344 TMM Trimmed Mean of M

345 AJCC American Joint Committee on Cancer 


$\begin{array}{lll}346 & \text { HR } & \text { Hazard ratio } \\ 347 & \text { CI } & \text { Confidence interval } \\ 348 & \text { GO } & \text { gene ontology } \\ 349 & \text { KEGG } & \text { Kyoto Encyclopedia of Genes and Genomes } \\ 350 & \text { DAVID } & \text { Database for Annotation, Visualization, and Integrated Discovery }\end{array}$

\section{References}

Blackstone EH. 2001. Breaking down barriers: helpful breakthrough statistical methods you need to understand better. J Thorac Cardiovasc Surg 122:430-439. 10.1067/mtc.2001.117536

Collins GS, Reitsma JB, Altman DG, and Moons KG. 2015. Transparent Reporting of a multivariable prediction model for Individual Prognosis Or Diagnosis (TRIPOD): the TRIPOD Statement. Br J Surg 102:148-158. 10.1002/bjs. 9736

Derrien T, Johnson R, Bussotti G, Tanzer A, Djebali S, Tilgner H, Guernec G, Martin D, Merkel A, Knowles DG, Lagarde J, Veeravalli L, Ruan X, Ruan Y, Lassmann T, Carninci P, Brown JB, Lipovich L, Gonzalez JM, Thomas M, Davis CA, Shiekhattar R, Gingeras TR, Hubbard TJ, Notredame C, Harrow J, and Guigo R. 2012. The GENCODE v7 catalog of human long noncoding RNAs: analysis of their gene structure, evolution, and expression. Genome Res 22:1775-1789. 10.1101/gr.132159.111

Fan Q, and Liu B. 2018. Discovery of a novel six-long non-coding RNA signature predicting survival of colorectal cancer patients. J Cell Biochem 119:3574-3585. 10.1002/jcb.26548

Ferlay J, Shin HR, Bray F, Forman D, Mathers C, and Parkin DM. 2010. Estimates of worldwide burden of cancer in 2008: GLOBOCAN 2008. Int J Cancer 127:2893-2917. 10.1002/ijc.25516

Grunkemeier GL, and Wu Y. 2004. Bootstrap resampling methods: something for nothing? Ann Thorac Surg 77:1142-1144. 10.1016/j.athoracsur.2004.01.005

Kita Y, Yonemori K, Osako Y, Baba K, Mori S, Maemura K, and Natsugoe S. 2017. Noncoding RNA and colorectal cancer: its epigenetic role. J Hum Genet 62:41-47. 10.1038/jhg.2016.66

Li Y, Xia Y, Li J, Wu D, Wan X, Wang K, Wu M, Liu J, Lau WY, and Shen F. 2015. Prognostic Nomograms for Pre- and Postoperative Predictions of Long-Term Survival for Patients Who Underwent Liver Resection for Huge Hepatocellular Carcinoma. J Am Coll Surg 221:962-974.e964. 10.1016/j.jamcollsurg.2015.08.003

McFadden EJ, and Hargrove AE. 2016. Biochemical Methods To Investigate lncRNA and the Influence of IncRNA:Protein Complexes on Chromatin. Biochemistry 55:1615-1630. 10.1021/acs.biochem.5b01141

Robinson MD, and Oshlack A. 2010. A scaling normalization method for differential expression analysis of RNAseq data. Genome Biol 11:R25. 10.1186/gb-2010-11-3-r25

Sanoff HK, Sargent DJ, Campbell ME, Morton RF, Fuchs CS, Ramanathan RK, Williamson SK, Findlay BP, Pitot HC, and Goldberg RM. 2008. Five-year data and prognostic factor analysis of oxaliplatin and irinotecan combinations for advanced colorectal cancer: N9741. $J$ Clin Oncol 26:5721-5727. $10.1200 /$ jco.2008.17.7147

Siegel RL, Miller KD, Fedewa SA, Ahnen DJ, Meester RGS, Barzi A, and Jemal A. 2017. Colorectal cancer statistics, 2017. CA Cancer J Clin 67:177-193. 10.3322/caac.21395 
400

401

402

403

404

405

406

407

408

409

410

411

412

413

414

415

416

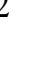

3

15

\section{6}

Siegel RL, Miller KD, and Jemal A. 2016. Cancer statistics, 2016. CA Cancer J Clin 66:7-30. 10.3322/caac.21332

Tian X, Zhu X, Yan T, Yu C, Shen C, Hong J, Chen H, and Fang JY. 2017. Differentially Expressed lncRNAs in Gastric Cancer Patients: A Potential Biomarker for Gastric Cancer Prognosis. J Cancer 8:2575-2586. 10.7150/jca. 19980

Weng M, Wu D, Yang C, Peng H, Wang G, Wang T, and Li X. 2017. Noncoding RNAs in the development, diagnosis, and prognosis of colorectal cancer. Transl Res 181:108-120. 10.1016/j.trsl.2016.10.001

Xie X, Tang B, Xiao YF, Xie R, Li BS, Dong H, Zhou JY, and Yang SM. 2016. Long non-coding RNAs in colorectal cancer. Oncotarget 7:5226-5239. 10.18632/oncotarget.6446

Xing Y, Zhao Z, Zhu Y, Zhao L, Zhu A, and Piao D. 2018. Comprehensive analysis of differential expression profiles of mRNAs and IncRNAs and identification of a 14-IncRNA prognostic signature for patients with colon adenocarcinoma. Oncol Rep 39:2365-2375. 10.3892/or.2018.6324

Xue W, Li J, Wang F, Han P, Liu Y, and Cui B. 2017. A long non-coding RNA expression signature to predict survival of patients with colon adenocarcinoma. Oncotarget 8:101298-101308. 10.18632/oncotarget.21064

Zeng JH, Liang L, He RQ, Tang RX, Cai XY, Chen JQ, Luo DZ, and Chen G. 2017. Comprehensive investigation of a novel differentially expressed lncRNA expression profile signature to assess the survival of patients with colorectal adenocarcinoma. Oncotarget 8:16811-16828. 10.18632/oncotarget.15161

(1)

(1)

(1)

3

(1)

5

6

7

8

9

0

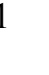




\section{Figure legends}

Figure 1. Study flowchart in the present study. TCGA, the Cancer Genome Atlas.

Figure 2. The nine-IncRNA prognostic signature to predict the overall survival of colorectal cancer patients

Figure 3. The distributions of nine-IncRNA prognostic signature (A), overall survival status and overall survival time $(B)$ in model cohort.

Figure 4. The Kaplan-Meier survival curves in model cohort.

432 Figure 5. Performance of nine-IncRNA prognostic signature in model cohort: Time-dependent receiver operating characteristic curves of three prognostic models according to 1-year (A), 3-year (B) and 5-year (C) overall survival. (D). Calibration curve for 1-year overall survival; (E). Calibration curve for 3-year overall survival; (F). Calibration curve for 5-year overall survival Figure 6. The distributions of nine-IncRNA prognostic signature $(A)$, overall survival status and overall survival time $(B)$ in validation cohort.

Figure 7. The Kaplan-Meier survival curves in validation cohort.

439 Figure 8. Performance of nine-IncRNA prognostic signature in validation cohort: Time-dependent 440 receiver operating characteristic curves of three prognostic models according to 1-year (A), 3-year (B) 441 and 5-year (C) overall survival. (D). Calibration curve for 1-year overall survival; (E). Calibration curve 442 for 3-year overall survival; (F). Calibration curve for 5-year overall survival 443 Figure 9. Functional enrichment analysis of prognostic signature. KEGG, Kyoto Encyclopedia of 
444 Genes and Genomes.

445

446

447

448

449

450

451 


\section{Figure 1}

\section{Study flowchart in the present study. TCGA, the Cancer Genome Atlas.}

The original data of model cohort from TCGA-COAD dataset contained 14,449 IncRNA expression information from 458 cancer samples and 41 adjacent normal tissues.

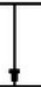

The differential gene expression analyses identified 574 potential IncRNAs $(n=458)$.
Colorectal cancer patients in model cohort were downloaded from the TCGA-COAD dataset $(n=629)$.

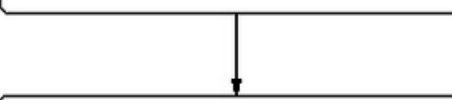

Patients with complete survival information $(n=580)$.

$+$

Patients with IncRNA information and complete survival information $(n=424)$.

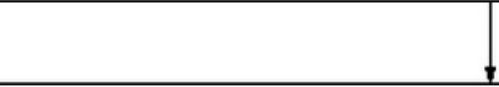

The multivariate cox regression identified 9 prognostic predictors for overall survival $(n=424)$.

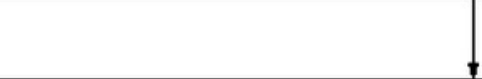

Final prognostic nomogram was constructed with 9 IncRNAs by using cox multivariate regression model (backward stepwise) $(n=424)$.

Survival curves, time-dependent receiver operating characteristic curves and calibration curves of nomogram $(n=424)$.

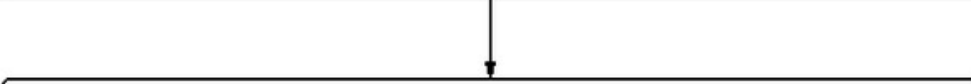

Internal validation by the bootstrap resample method. The validation cohort $(n=424)$ was generated by random drawing from the original model cohort $(n=424)$ with replacement method.

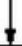

Survival curves, time-dependent receiver operating characteristic curves and calibration curves of nomogram in validation cohort $(n=424)$. 
Figure 2

The nine-IncRNA prognostic signature to predict the overall survival of colorectal cancer patients

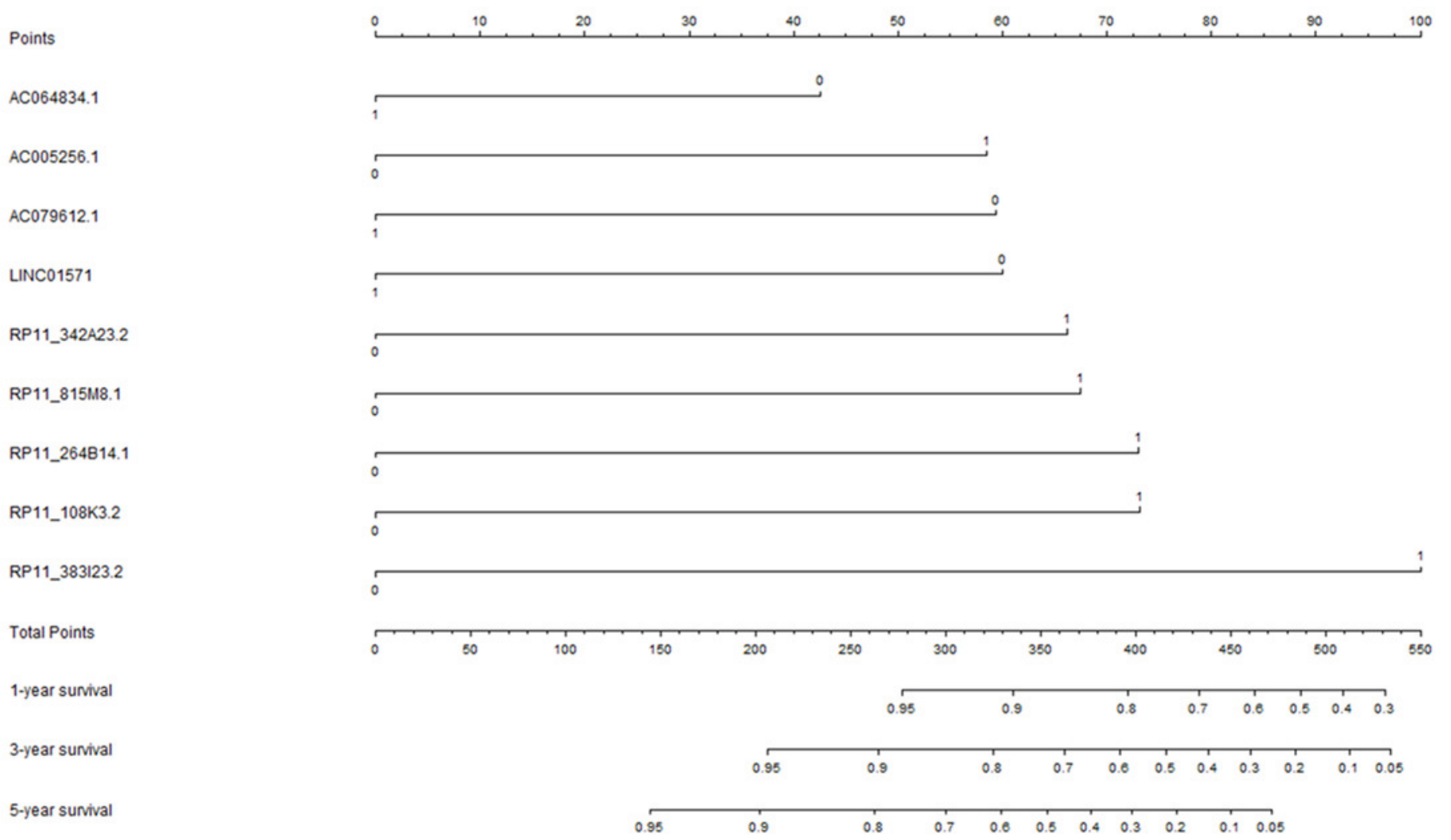


Figure 3

The distributions of nine-IncRNA prognostic signature (A), overall survival status and overall survival time (B) in model cohort.

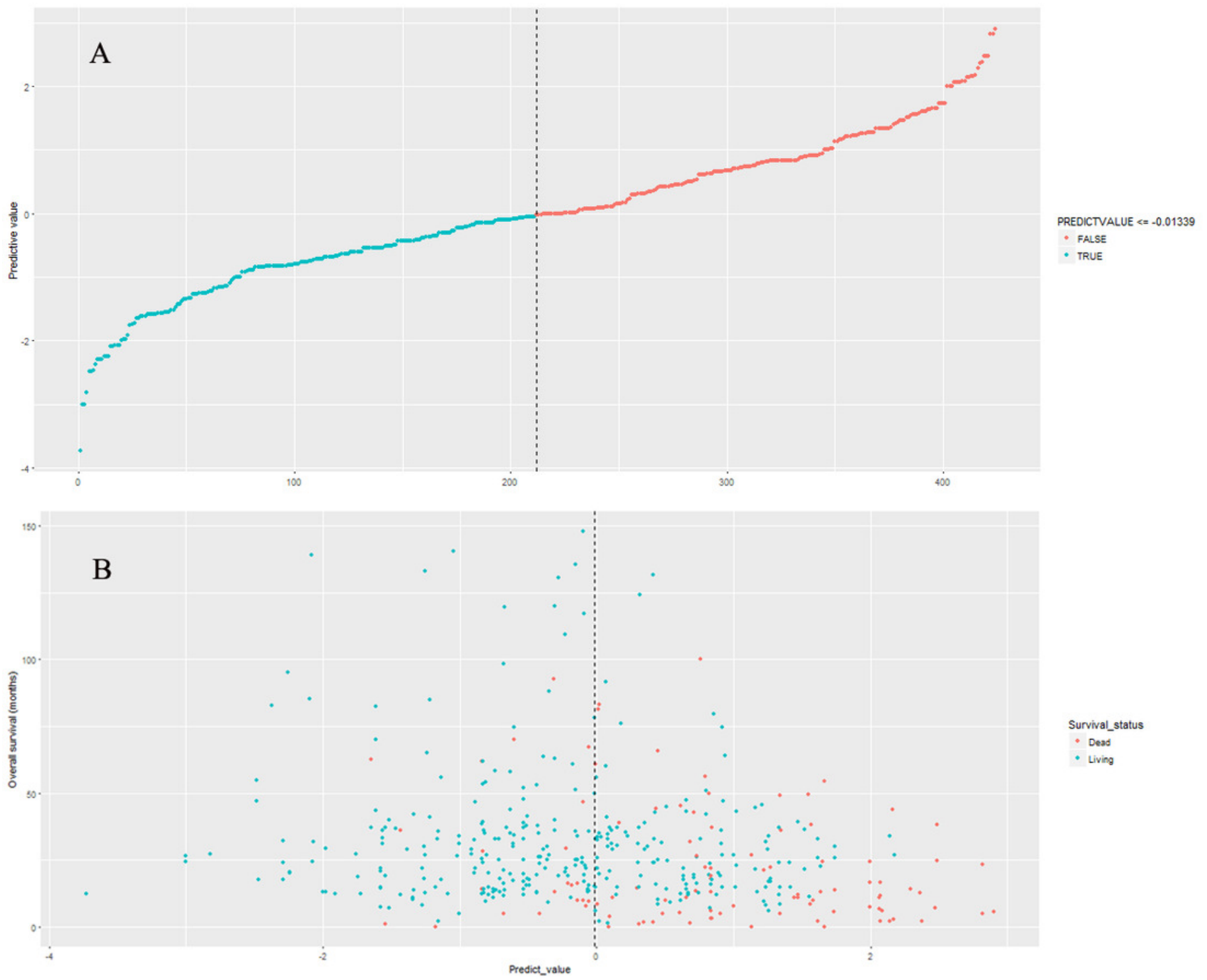


Figure 4

The Kaplan-Meier survival curves in model cohort.

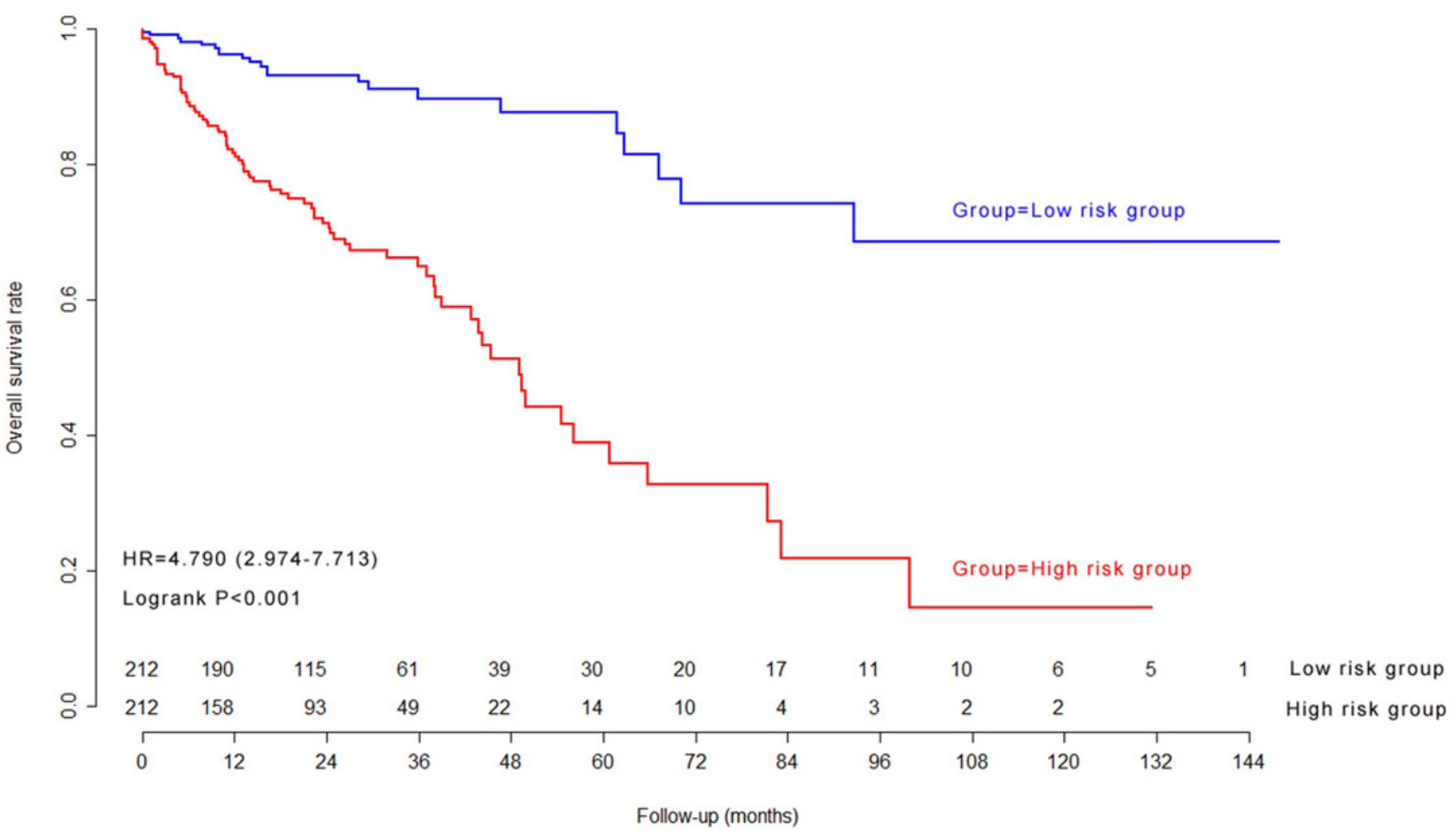


Figure 5

Performance of nine-IncRNA prognostic signature in model cohort: Time-dependent receiver operating characteristic curves of three prognostic models according to 1-year (A), 3-year (B) and 5-year (C) overall survival. (D). Calibration curve for 1
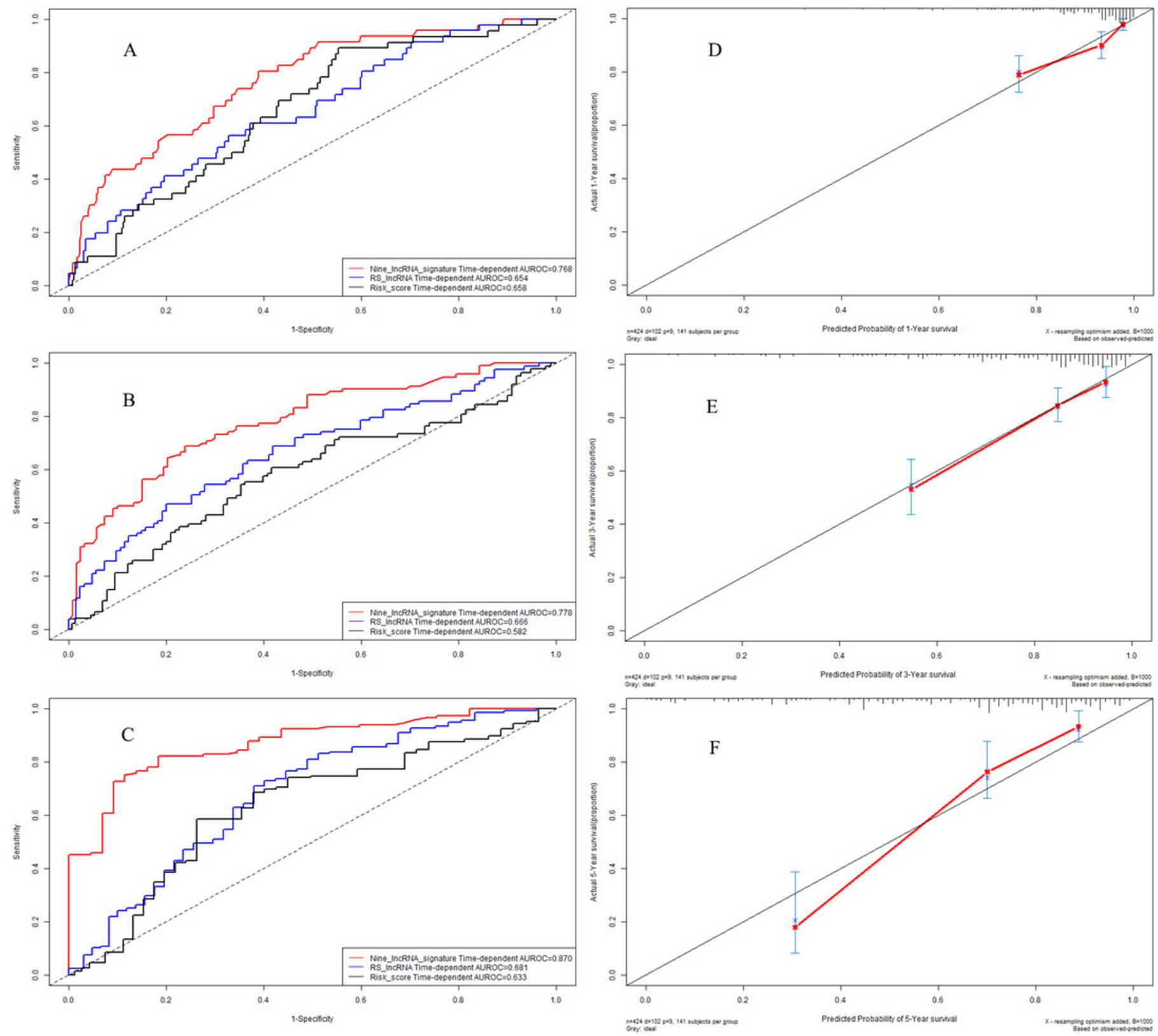
Figure 6

The distributions of nine-IncRNA prognostic signature (A), overall survival status and overall survival time $(B)$ in validation cohort

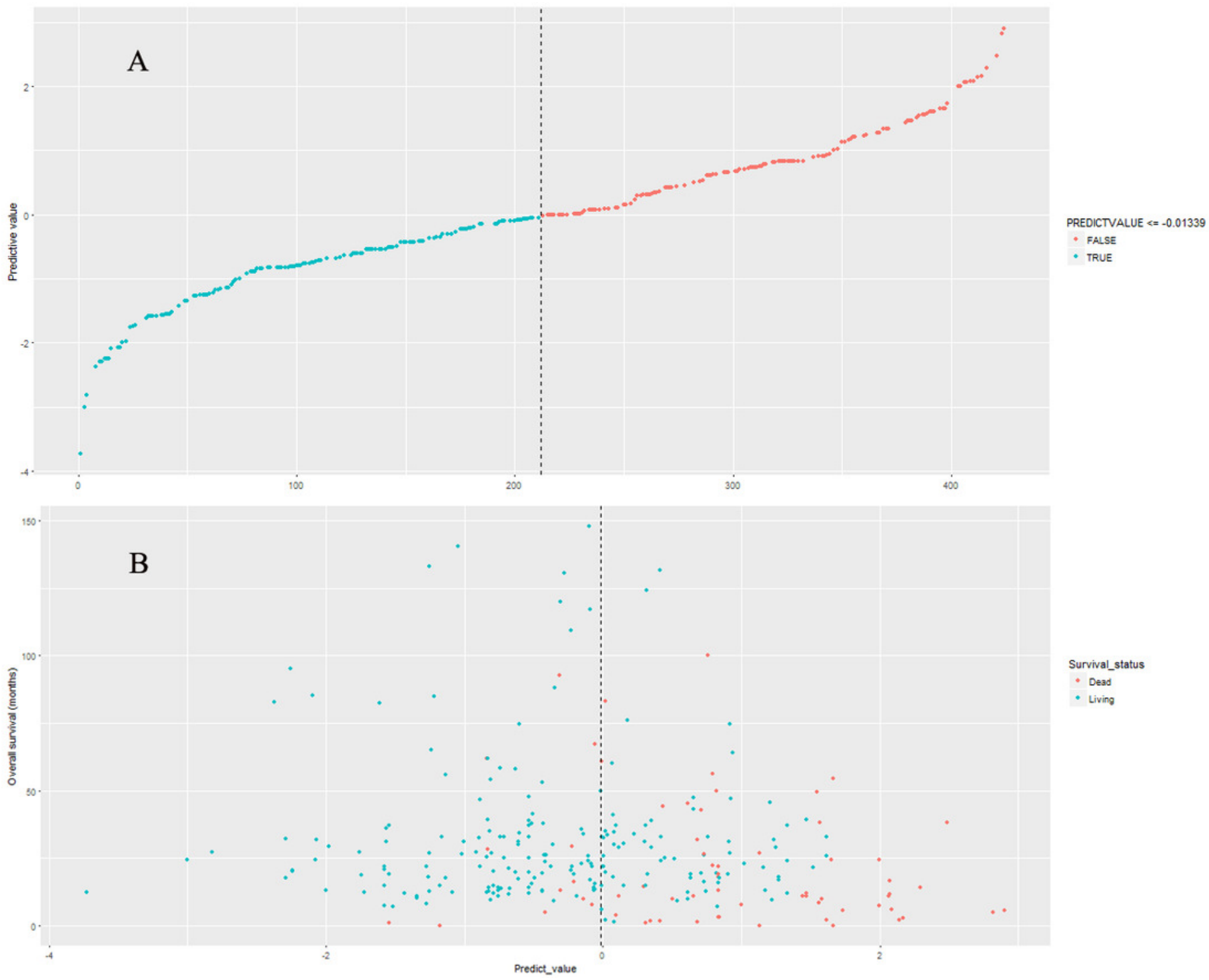


Figure 7

The Kaplan-Meier survival curves in validation cohort

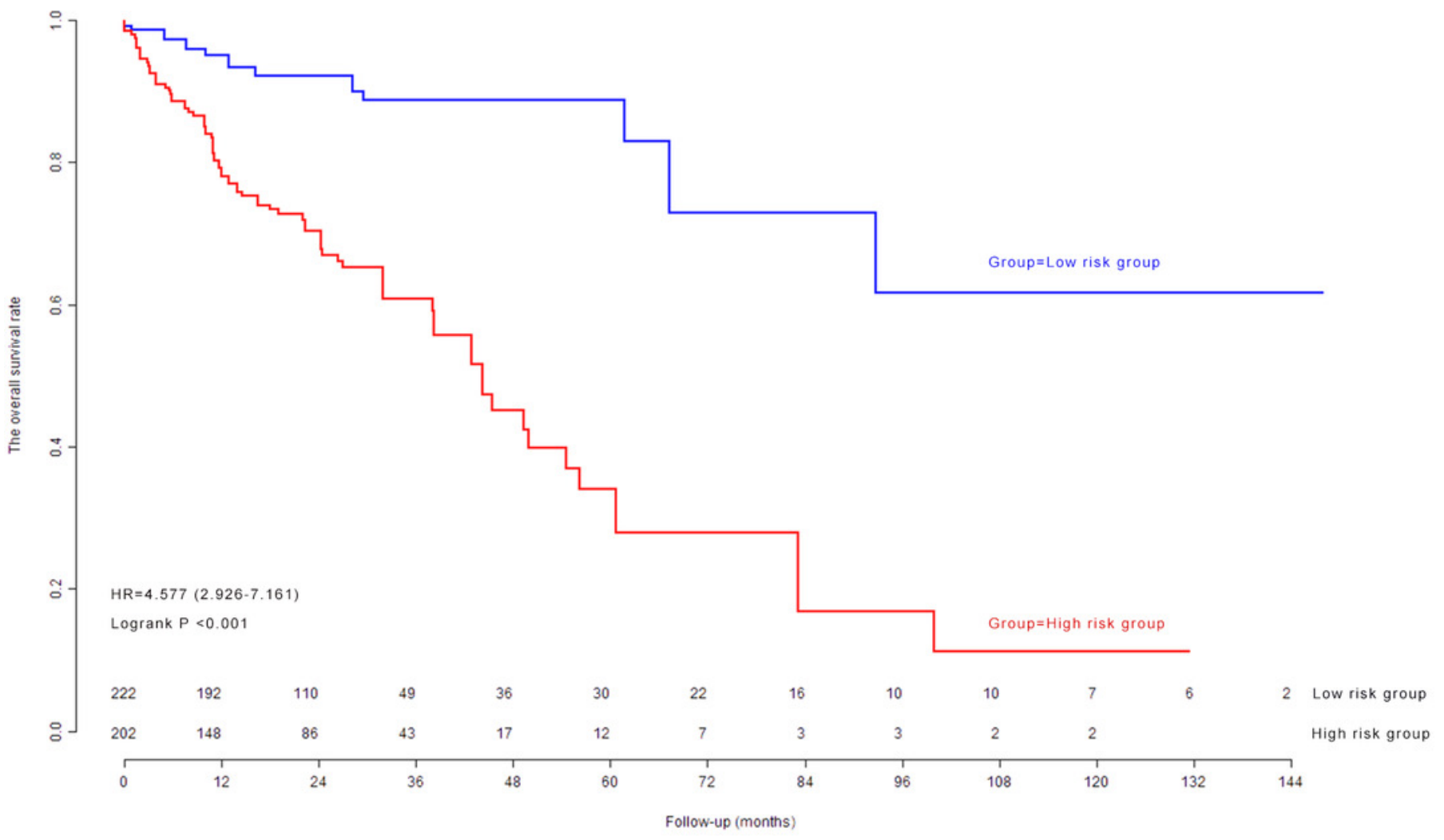


Figure 8

Performance of nine-IncRNA prognostic signature in validation cohort: Time-dependent receiver operating characteristic curves of three prognostic models according to 1-year (A), 3-year (B) and 5-year (C) overall survival. (D). Calibration curve
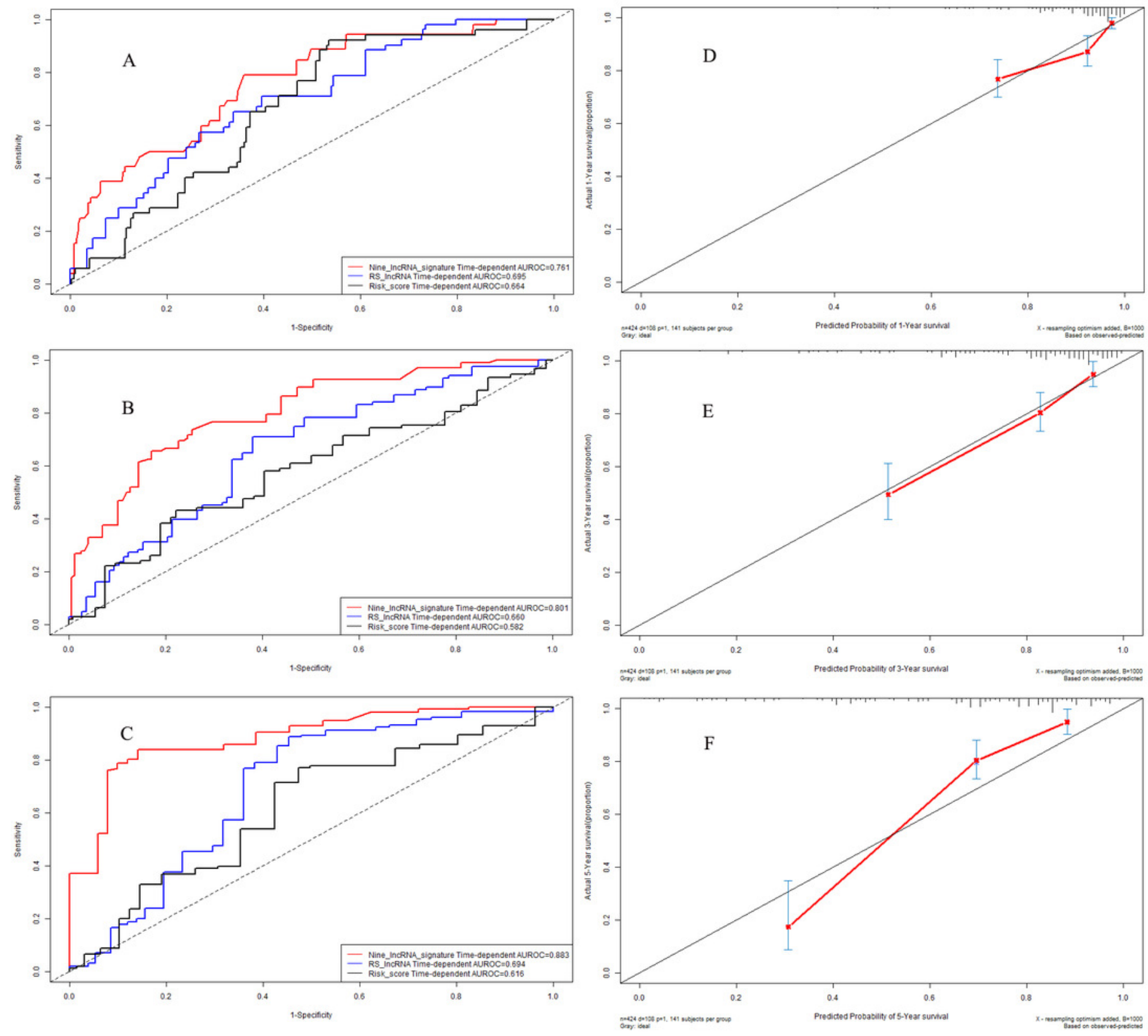


\section{Figure 9}

\section{Functional enrichment analysis of prognostic signature. KEGG, Kyoto Encyclopedia of Genes and Genomes}

\section{A.Biological Process}

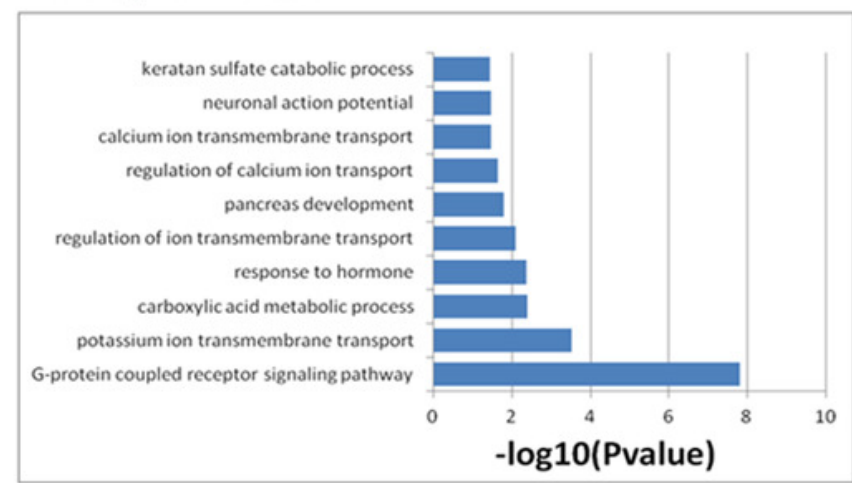

\section{C.Cellular Components}

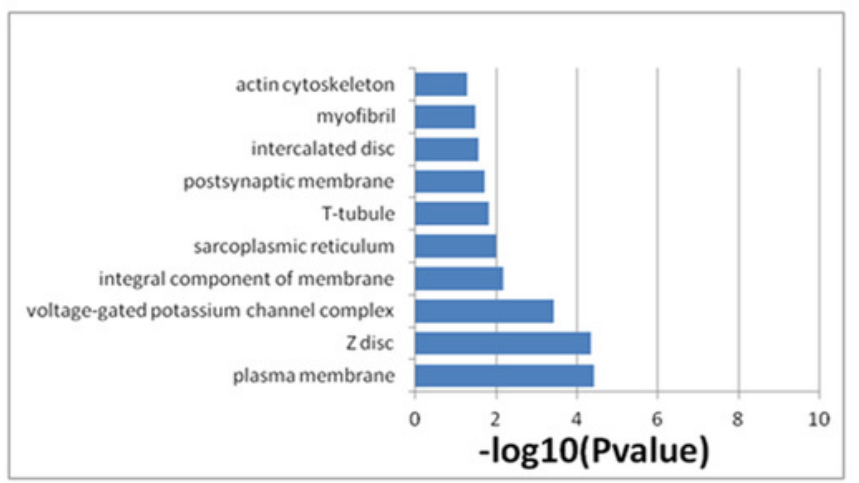

\section{B. Molecular Function}

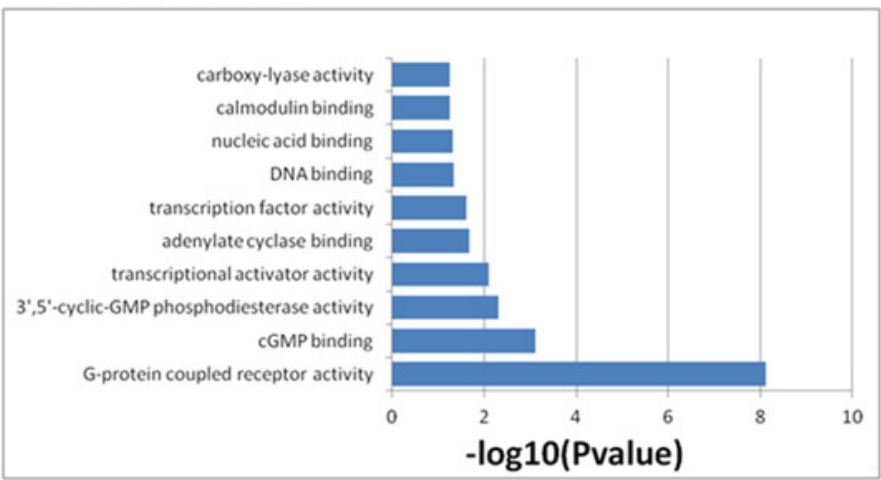

D. KEGG Pathway

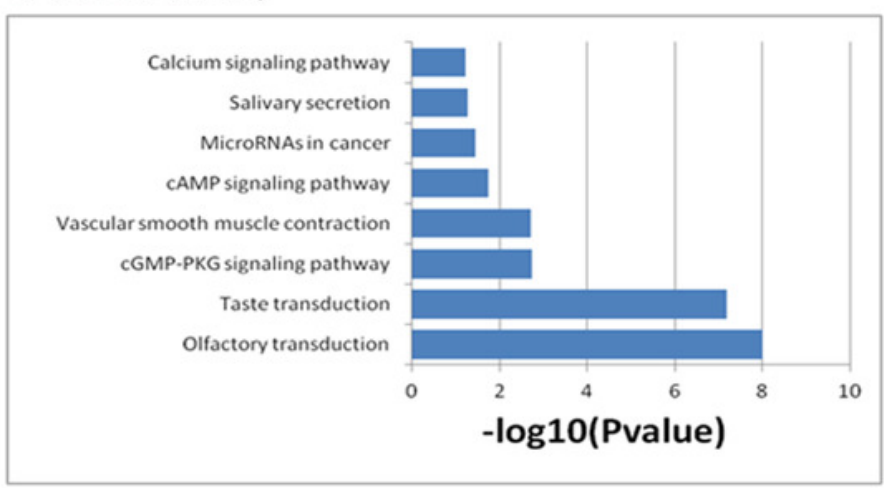




\section{Table $\mathbf{1}$ (on next page)}

The clinical features of colorectal cancer patients in model cohort and validation cohort 
1 Table 1. The clinical features of colorectal cancer patients in model cohort and validation cohort

2

\begin{tabular}{|c|c|c|c|}
\hline & Model cohort $(n=424)$ & Validation cohort $(n=424)$ & $P$ value \\
\hline Death [n(\%)] & $102(24.0)$ & $108(25.5)$ & 0.633 \\
\hline Survival time (mean $\pm \mathrm{SD}$, month) & $30.0 \pm 25.5$ & $28.6 \pm 25.4$ & 0.163 \\
\hline Age (mean $\pm S D$, year) & $66.7 \pm 13.0$ & $67.1 \pm 12.4$ & 0.612 \\
\hline Gender (Male/Female) & $230 / 194$ & $224 / 200$ & 0.680 \\
\hline \multicolumn{4}{|l|}{ Tumor site } \\
\hline Colon & $361(85.1)$ & $351(82.8)$ & 0.363 \\
\hline Rectum & $58(13.7)$ & $69(16.3)$ & \\
\hline NR & $5(1.2)$ & $4(0.9)$ & \\
\hline AJCC Stage (IV/III/II/I/NA) & $59 / 124 / 162 / 68 / 11$ & $58 / 145 / 143 / 68 / 10$ & 0.578 \\
\hline AJCC PT (T4/T3/T2/T1/NA) & $51 / 292 / 70 / 11 / 0$ & $54 / 286 / 73 / 11 / 0$ & 0.976 \\
\hline AJCC PN (N2/N1/N0/NA) & $77 / 102 / 245 / 0$ & $88 / 105 / 231 / 0$ & 0.756 \\
\hline AJCC PM (MX/M1/M0/NA) & $47 / 59 / 312 / 6$ & $46 / 58 / 314 / 6$ & 0.998 \\
\hline Radiation treatment adjuvant (Yes/No /NA) & $0 / 33 / 391$ & $0 / 42 / 382$ & 0.553 \\
\hline Pharmaceutial adjuvant (Yes/No /NA) & $18 / 15 / 391$ & $22 / 20 / 382$ & 0.608 \\
\hline History other malignance (Yes/No /NA) & $56 / 368 / 0$ & $50 / 374 / 0$ & 0.533 \\
\hline AC005256.1 (High/Low) & $212 / 212$ & $212 / 212$ & 1.0 \\
\hline RP11_815M8.1 (High/Low) & $212 / 212$ & $219 / 205$ & 0.631 \\
\hline RP11_342A23.2 (High/Low) & $212 / 212$ & $212 / 212$ & 1.0 \\
\hline RP11_264B14.1 (High/Low) & $212 / 212$ & $196 / 228$ & 0.272 \\
\hline AC064834.1 (High/Low) & $212 / 212$ & $215 / 209$ & 0.837 \\
\hline RP11_108K3.2 (High/Low) & $212 / 212$ & $207 / 217$ & 0.731 \\
\hline LINC01571 (High/Low) & $212 / 212$ & $206 / 218$ & 0.680 \\
\hline RP11_383I23.2 (High/Low) & $212 / 212$ & $206 / 218$ & 0.680 \\
\hline AC079612.1 (High/Low) & $212 / 212$ & $237 / 187$ & 0.085 \\
\hline
\end{tabular}

3 Note: Continuous variables were compared by t-test or Mann-Whitney $U$ test as appropriate.

4 Categorical variables were compared by chi-squared test or Fisher's exact test as appropriate. NA, 5 missing data. NR, not reported.

6 


\section{Table 2 (on next page)}

The overall information of nine prognostic IncRNA predictors in univariate and multivariable Cox regression analyses

Note: HR, hazard ratio; Cl, confidence interval. The medians of IncRNA expression values

were used as cutoff values to stratify IncRNA expression values into high expression group (as value 1 ) and low expression group (as value 0 ). 
1 Table 2. The overall information of nine prognostic IncRNA predictors in univariate and multivariable 2 Cox regression analyses

3

\begin{tabular}{|c|c|c|c|c|c|c|c|}
\hline & \multicolumn{3}{|c|}{ Univariate analysis } & \multicolumn{4}{|c|}{ Multivariate analysis } \\
\hline & $\mathrm{HR}$ & $95 \% \mathrm{Cl}$ & $P$-value & coefficient & $\mathrm{HR}$ & $95 \% \mathrm{Cl}$ & $P$-value \\
\hline RP11_815M8.1 (High/Low) & 1.842 & $1.232-2.754$ & 0.003 & 0.837 & 2.310 & $1.473-3.623$ & $<0.001$ \\
\hline RP11_342A23.2 (High/Low) & 1.580 & $1.063-2.347$ & 0.024 & 0.822 & 2.276 & $1.490-3.477$ & $<0.001$ \\
\hline RP11_264B14.1 (High/Low) & 1.510 & $1.015-2.246$ & 0.042 & 0.905 & 2.471 & $1.582-3.857$ & $<0.001$ \\
\hline AC064834.1 (High/Low) & 0.590 & $0.396-0.880$ & 0.010 & -0.529 & 0.589 & $0.391-0.889$ & 0.012 \\
\hline RP11_108K3.2 (High/Low) & 2.552 & $1.659-3.927$ & $<0.001$ & 0.907 & 2.478 & $1.592-3.855$ & $<0.001$ \\
\hline LINC01571 (High/Low) & 0.661 & $0.446-0.981$ & 0.040 & -0.745 & 0.475 & $0.307-0.733$ & $<0.001$ \\
\hline RP11_383I23.2 (High/Low) & 1.667 & $1.117-2.487$ & 0.012 & 1.241 & 3.459 & $2.143-5.583$ & $<0.001$ \\
\hline AC079612.1 (High/Low) & 0.450 & $0.297-0.681$ & $<0.001$ & -0.737 & 0.479 & $0.314-0.731$ & $<0.001$ \\
\hline AC005256.1 (High/Low) & 1.606 & $1.080-2.387$ & 0.019 & 0.725 & 2.065 & $1.352-3.155$ & $<0.001$ \\
\hline
\end{tabular}

4 Note: $\mathrm{HR}$, hazard ratio; $\mathrm{Cl}$, confidence interval. The medians of IncRNA expression values were used 5 as cutoff values to stratify IncRNA expression values into high expression group (as value 1) and low 6 expression group (as value 0 ).

7

8

9

10

11

12

13

14

15

16

17

18

19

20

21

22

23

24

25

26

27

28

29

30 


\section{Table 3(on next page)}

Univariate and multivariable Cox regression analyses

Note: AJCC, the American Joint Committee on Cancer; HR, hazard ratio; $\mathrm{Cl}$, confidence interval. The median of nine-IncRNA prognostic signature score was used as the cutoff value to stratify colorectal patients into high risk group and low risk group 
2 Table3. Univariate and multivariable Cox regression analyses

3

\begin{tabular}{|c|c|c|c|c|c|c|c|c|}
\hline & \multirow[b]{2}{*}{$\mathrm{n}$} & \multicolumn{2}{|c|}{ Univariate analysis } & \multirow[b]{2}{*}{$P$-value } & \multicolumn{2}{|c|}{ Multivariate analysis } & \multirow[b]{2}{*}{$95 \% \mathrm{Cl}$} & \multirow[b]{2}{*}{$P$-value } \\
\hline & & $\mathrm{HR}$ & $95 \% \mathrm{Cl}$ & & coefficient & $\mathrm{HR}$ & & \\
\hline \multicolumn{9}{|l|}{ Model cohort $(n=424)$} \\
\hline Age(year) & 424 & 1.022 & $1.005-1.038$ & 0.010 & 0.025 & 1.025 & $1.007-1.044$ & 0.007 \\
\hline Gender (Male/Female) & 424 & 1.112 & $0.752-1.645$ & 0.595 & -0.101 & 0.904 & $0.595-1.373$ & 0.636 \\
\hline AJCC PT $(T 4, T 3 / T 2, T 1)$ & 424 & 2.926 & $1.356-6.318$ & 0.006 & 1.161 & 3.193 & $1.248-8.165$ & 0.015 \\
\hline AJCC PN (N2,N1/N0) & 424 & 2.506 & $1.683-3.733$ & $<0.001$ & -0.551 & 0.576 & $0.218-1.524$ & 0.267 \\
\hline AJCC PM (MX,M1/M0) & 418 & 2.962 & $1.985-4.419$ & $<0.001$ & 0.707 & 2.029 & $1.284-3.205$ & 0.002 \\
\hline AJCC stage $(I V, I I I / I I, I)$ & 413 & 2.841 & $1.869-4.317$ & $<0.001$ & 1.233 & 3.433 & $1.157-10.187$ & 0.026 \\
\hline Nine-IncRNA prognostic signature (High/Low) & 424 & 4.790 & $2.974-7.713$ & $<0.001$ & 1.513 & 4.539 & $2.722-7.571$ & $<0.001$ \\
\hline \multicolumn{9}{|l|}{ Validation cohort $(n=424)$} \\
\hline Age(year) & 424 & 1.017 & $1.002-1.033$ & 0.032 & 0.031 & 1.031 & $1.012-1.050$ & 0.001 \\
\hline Gender (Male/Female) & 424 & 1.051 & $0.720-1.535$ & 0.797 & -0.213 & 0.808 & $0.538-1.214$ & 0.306 \\
\hline AJCC PT $(\mathrm{T} 4, \mathrm{~T} 3 / \mathrm{T} 2, \mathrm{~T} 1)$ & 424 & 8.353 & $2.646-26.368$ & $<0.001$ & 3.079 & 21.729 & $2.988-158.025$ & 0.002 \\
\hline AJCC PN (N2,N1/N0) & 424 & 2.253 & $1.528-3.323$ & $<0.001$ & -0.526 & 0.591 & $0.260-1.346$ & 0.211 \\
\hline AJCC PM (MX,M1/M0) & 418 & 2.347 & $1.577-3.493$ & $<0.001$ & 0.570 & 1.769 & $1.107-2.827$ & 0.017 \\
\hline AJCC stage (IV,III/II,I) & 414 & 2.741 & $1.817-4.134$ & $<0.001$ & 1.231 & 3.425 & 1.317-8.905 & 0.012 \\
\hline Nine-IncRNA prognostic signature (High/Low) & 424 & 4.577 & $2.926-7.161$ & $<0.001$ & 1.515 & 4.551 & $2.806-7.383$ & $<0.001$ \\
\hline
\end{tabular}

4 Note: AJCC, the American Joint Committee on Cancer; HR, hazard ratio; Cl, confidence interval. The 5 median of nine-IncRNA prognostic signature score was used as the cutoff value to stratify colorectal 6 patients into high risk group and low risk group.

7 
